\title{
Converting Insulin-like Growth Factors 1 and 2 into High-Affinity Ligands for Insulin Receptor Isoform A by the Introduction of an Evolutionarily Divergent Mutation
}

Kateřina Macháčková, ${ }^{\dagger}$ Martina Chrudinová, ${ }^{\dagger}$ Jelena Radosavljević, ${ }^{\dagger, \|}$ Pavlo Potalitsyn, Květoslava Kř́žžnova, ${ }^{\dagger}$ Milan Fábry, ${ }^{\ddagger}$ Irena Selicharová, ${ }^{\dagger}$ Michaela Collinsová, ${ }^{\dagger}$ Andrzej M. Brzozowski, ${ }^{\S}$

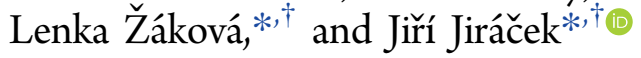

\footnotetext{
${ }^{\dagger}$ Institute of Organic Chemistry and Biochemistry, The Czech Academy of Sciences, Flemingovo nám 2, 16610 Prague 6, Czech Republic

${ }^{\ddagger}$ Institute of Molecular Genetics, The Czech Academy of Sciences, Flemingovo n. 2, 16637 Prague 6, Czech Republic

${ }^{\S}$ York Structural Biology Laboratory, Department of Chemistry, The University of York, Heslington, York YO10 5DD, United Kingdom
}

Supporting Information

ABSTRACT: Insulin-like growth factors 1 and 2 (IGF-1 and -2, respectively) are protein hormones involved not only in normal growth and development but also in life span regulation and cancer. They exert their functions mainly through the IGF-1R or by binding to isoform A of the insulin receptor (IR-A). The development of IGF-1 and IGF-2 antagonists is of great clinical interest. Mutations of A4 and A8 sites of human insulin lead to disproportionate effects on hormone IR binding and activation. Here, we systematically modified IGF-1 sites 45, 46, and 49 and IGF-2 sites 45 and 48, which correspond, or are close, to insulin sites A4 and A8. The IGF-1R and IR-A binding and autophosphorylation potencies of these analogues were characterized. They retained the main IGF-1R-related properties, but the hormones with His49 in IGF-1 and His48 in IGF-2 showed significantly higher affinities for IR-A and for IR-B, being the strongest IGF-1- and IGF-2-like binders of these receptors ever reported. All analogues activated IR-A and IGF-1R without major discrepancies in their binding affinities. This study revealed that IR-A and IGF-1R contain specific sites, likely parts of their so-called sites 2 ', which can interact differently with specifically modified IGF analogues. Moreover, a clear importance of IGF-2 site 44 for effective hormone folding was also observed. These findings may facilitate novel and rational engineering of new hormone analogues for IR-A and IGF-1R studies and for potential medical applications.

$\mathrm{T}$ wo insulin-like growth factors, IGF-1 and IGF-2, together with insulin, are members of a family of small protein hormones that share common evolutionary origins, ${ }^{1-4}$ having similar primary (Figure 1) and three-dimensional structures. They regulate a wide spectrum of key physiological events, with insulin being responsible mainly for broad, metabolic control, ${ }^{6}$ while IGF-1 and IGF-2 are growth factors involved primarily in the development and growth of mammals. ${ }^{5}$ The role of IGF-1 is relatively well studied, ${ }^{7-9}$ but physiological functions of IGF2 are much less understood, ${ }^{10}$ despite emerging evidence of its impact on the central nervous system. ${ }^{11,12}$

IGF-1, IGF-2, and insulin exert their activities by binding to different but highly homologous $(\sim 75 \%), \sim 450 \mathrm{kDa}(\alpha \beta)_{2}$ dimeric tyrosine-kinase receptors: IGF-1 receptor (IGF-1R) and insulin receptor (IR). ${ }^{13-15}$ IR exists in two isoforms, IR-A, and IR-B, with distinct biochemical properties ${ }^{16,17}$ and a specific tissue distribution. IR-B is a predominant IR form in liver, while muscle and adipose tissues contain both isoforms at different ratios. IR-A is predominant in brain, fetus, and lymphatic tissues and is considered mainly as a "mitogenic" form of the IR, in contrast to IR-B that is considered as the main "metabolic" receptor for insulin. ${ }^{16-18}$ A high degree of homology of these receptors results in a significant crossbinding of insulin and both IGFs to IR-A and IGF-1R, ${ }^{19}$ and hence some overlapping biological responses to binding of these ligands. $^{20,21}$

The binding of insulin and IGFs to these receptors triggers two major signaling pathways that are initiated by the autophosphorylation of tyrosines within their intracellular tyrosine kinase domains. ${ }^{22}$ The phosphoinositide 3-kinase (PI3K)/Akt pathway leads to the metabolic, glycemic responses and effects of the hormone:receptor complex, but it is also important for growth and protein synthesis. ${ }^{23}$ The Ras/ERK main pathway involves activation of the Ras/Raf/MAPK/ ERK1/2 cascade, which mediates proliferative effects through gene transcription regulation. ${ }^{21}$ Whereas insulin signals mainly via both IR isoforms, ${ }^{24}$ IGF-1 and IGF-2 promote mitogenic

Received: December 15, 2017

Revised: March 29, 2018

Published: April 2, 2018 


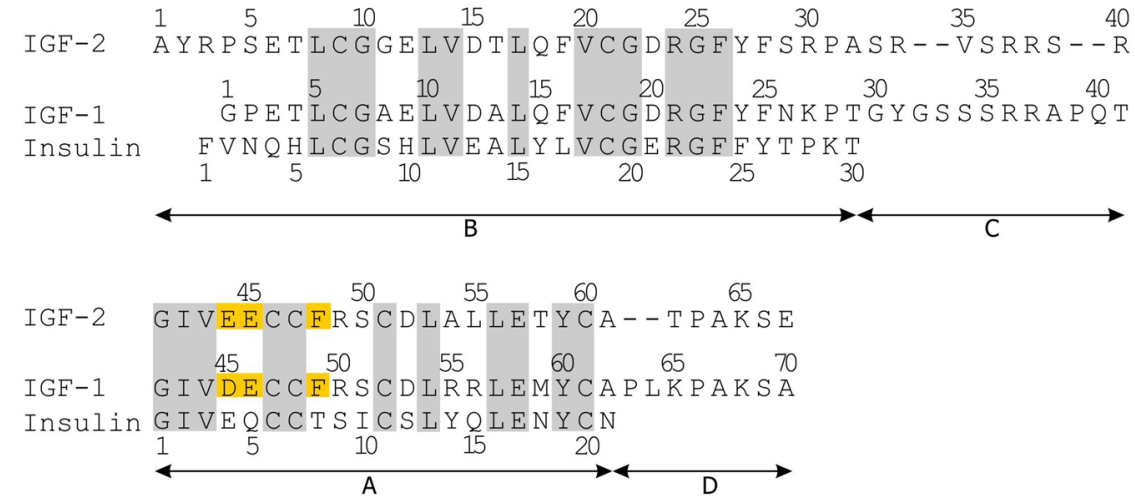

Figure 1. Comparison of the primary sequences of human IGF-1, IGF-2, and insulin. The organization of IGF-1 and IGF-2 into B, C, A, and D domains is shown below the sequences. Insulin A and B chains correspond to IGF A and B domains, respectively. The homologous regions are highlighted in gray, and the residues mutated in this study are highlighted in yellow.
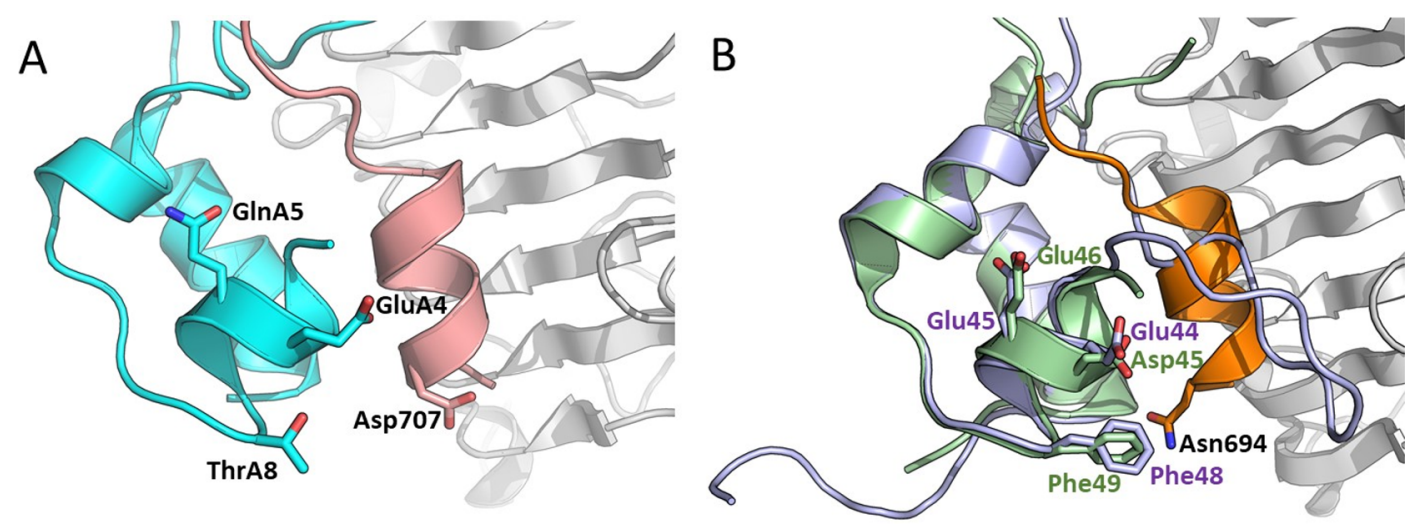

Figure 2. Receptor-bound structures of human insulin and human IGF-1 overlaid with human IGF-2. (A) Complex (4OGA) ${ }^{38}$ of human insulin sitting on the IR-L1 domain and IR $\alpha$-CT peptide. Insulin is colored cyan, the L1 domain light gray, and $\alpha$-CT pink. Side chains of insulin residues GluA4, GlnA5, ThrA8, and $\alpha$-CT Asp707 are shown and labeled. (B) Complex (4XSS) ${ }^{39}$ of human IGF-1 (violet) bound to the IR-L1 domain (light gray) and the IGF-1R $\alpha$-CT peptide (orange). Side chains of IGF-1 residues Asp45, Glu46, and Phe49 mutated in this study and $\alpha$-CT Asn694 are shown and labeled. The complex is overlaid with the NMR structure of human IGF-2 (5L3L), ${ }^{40}$ which is colored light green. Mutated IGF-2 residues Glu44, Glu45, and Phe48 and $\alpha$-CT Asp694 are also shown and labeled.

signaling through IGF-1R, and, importantly, similar mitogenic stimulation may result from binding of IGF-2 to IR-A. ${ }^{25}$ The complexity of insulin/IGF signaling is amplified further by the heterodimerization of IGF-1R and IR-A, and the presence of hybrid receptors that can be effectively activated by IGF-1, but not by insulin. ${ }^{26,27}$ Moreover, the bioavailability of free IGF-1 and IGF-2 for receptor signaling is modulated by a family of high-affinity IGF binding proteins 1-6 (IGFBP 1-6, respectively), ${ }^{28,29}$ and the circulation level of IGF-2 is also affected by a structurally distinct, and presumably nonsignaling, insulin-like growth factor type 2 receptor (IGF-2R), also known as the mannose 6-phosphate receptor. ${ }^{30}$ The equilibrium of all individual components and the appropriate function of the entire insulin/IGF system are essential for a proper functioning of the organism. ${ }^{31}$

In recent years, the role of the IGF/insulin system in cancer development and growth has been widely studied. ${ }^{10,31,32}$ Substantial efforts have been focused on the development of new anti-IGF-1R-directed therapies, mostly tyrosine-kinase (TK) inhibitors and antireceptor antibodies. ${ }^{33}$ However, the results of clinical trials were not satisfactory, ${ }^{34}$ because of either the toxicity of the TK-targeting drugs or an increasing overlap and takeover of IGF-1R signaling pathways by the IR. The lack of progress in addressing one of the key hallmarks of cancer underlines the need for new anticancer therapies that would exploit alternative, and specific, targets of the insulin/IGF axis. Here, a high-affinity/no-efficacy IGF-based IGF-1 analogue, i.e., selective antagonist of the IGF-1R, should represent a promising new strategy for combating IGF-1R-related malignancies.

To date, no IGF-like peptide antagonists of the IGF-1R have been identified. However, peptides with good IR/IGF-1R binding and antagonistic properties toward these receptors were discovered by a phage-display technique. ${ }^{35,36}$ Whittaker et al. ${ }^{37}$ showed that a combination of GluA4His and ThrA8His mutations of human insulin results in insulin analogues with native IR binding affinity but poor efficacy, an impaired ability to stimulate autophosphorylation of IR, and downstream Akt activation. They also proposed that surfaces involving insulin GluA4 and IR Asp707 could be behind the mechanism of receptor activation.

Interestingly, insulin acidic GluA4 is preserved by its equivalent Asp45 and Glu44 in IGF-1 and IGF-2, respectively (Figure 1). However, insulin-neutral Gln A5 is replaced by Glu46, and Glu45 in corresponding sites of IGF-1 and IGF-2. In addition, Asp707 of IR $\alpha$-CT is replaced by a neutral Asn694 in the IGF-1R $\alpha$-CT segment (Figure 2).

These correlations and trends between positions A4 and A5 in insulin and their IGFs equivalent 45 and 46 (in IGF-1) and 44 and 55 (in IGF-2) sites prompted us to study (i) the impact 
of the mutations of these residues on receptor affinities and potencies of these hormones, and (ii) whether such mutations can generate significant IGF-1R-specific antagonists, with potential anticancer clinical applications. A series of 14 IGF-1 and IGF-2 analogues mutated at these sites have been designed and made, and their binding to IGF-1R and IR-A and abilities to activate the receptors were characterized. Some of these mutations were also combined with the Phe49His mutation in IGF-1 and the Phe48His mutation in IGF-2, as it has been shown that insulin-corresponding mutation ThrA8His significantly increased the IR-A binding potency of this analogue. ${ }^{37,41-46}$

Despite the results for the analogues studied here not showing any antagonism, they revealed interesting properties of new IGF-1 and IGF-2 mutants that can interact differently with receptors for insulin and IGF-1. This could indicate new directions for a rational engineering of these hormones.

\section{MATERIALS AND METHODS}

Cloning and Production of IGF-1 and IGF-2 Analogues. As in our previously published research, ${ }^{40}$ both human IGF-1 (UniprotKB entry P05019 amino acids 49-118) and human IGF-2 (UniprotKB entry P01344 amino acids 25-91) have been cloned into a modified pRSFDuet-1 expression vector (kindly provided by E. Bouřa from the Institute of Organic Chemistry and Biochemistry in Prague) as a fusion with an N-terminally His6-tagged GB1 protein and TEV protease cleavage site. An additional $\mathrm{N}$-terminal glycine residue (Gly-1) was incorporated into IGF-1 to enable cleavage by TEV protease (Glu-Asn-Leu-Tyr-Phe-Gln $\downarrow$ Gly). In contrast to our previous study, ${ }^{40}$ the TEV protease cleavage site for the IGF-2 expression construct was modified to Glu-Asn-Leu-TyrPhe-Gln $\downarrow$ Ala, cleavage $(\downarrow)$ of which resulted in a native hormone with an N-terminal alanine. [D45H], [D45N], $[\mathrm{D} 45 \mathrm{~A}],[\mathrm{E} 46 \mathrm{H}],[\mathrm{E} 46 \mathrm{Q}]$, and [E46A] mutations in IGF-1 analogues were obtained by the standard site-directed mutagenesis protocol $(\mathrm{SDM})^{47}$ using appropriate mutagenic primers (listed in Table S1). After sequence verification, the mutant fragments were reintroduced into the expression vector. Additional [D45N+E46Q], [F49H], [E46H+F49H], [E46Q $+\mathrm{F} 49 \mathrm{H}]$, and $[\mathrm{D} 45 \mathrm{~N}+\mathrm{E} 46 \mathrm{Q}+\mathrm{F} 49 \mathrm{H}]$ mutations in IGF-1 analogues and in all cloned IGF-2 analogues were introduced either by the overlap-extension polymerase chain reaction (OE strategy in Table S1), ${ }^{48}$ using specific primers as flanking master primers and subsequent recloning into expression vector, or by the standard site-directed mutagenesis as mentioned above (SDM strategy in Table S1), using appropriate mutagenic primers.

We succeeded in expressing and purifying all planned IGF-1 analogues. However, only three IGF-2 analogues (with mutations [E45Q], $[\mathrm{F} 48 \mathrm{H}]$, and $[\mathrm{E} 45 \mathrm{Q}+\mathrm{F} 48 \mathrm{H}])$ were successfully produced. A list of successfully expressed constructs along with primers used for mutagenesis is provided as Table S1.

All successfully constructed analogues were produced, purified, and characterized, using the procedures described by Hexnerova et al. ${ }^{40}$ The purity of all tested analogues was $>95 \%$ (and controlled by reverse phase high-performance liquid chromatography analyses and high-resolution mass spectrometry spectra).

Biological Characterization of IGF-1 and IGF-2 Analogues. Binding affinities for the receptors were determined with receptors in the intact cells. Specifically, binding affinities for IGF-1R were determined with mouse fibroblasts transfected with human IGF-1R and with deleted mouse IGF-1R according to Hexnerova et al. ${ }^{40}$ Binding affinities for IR-A were determined with human IR-A in human IM-9 lymphocytes according to Vikova et al. ${ }^{49}$ Binding affinities for IR-B were determined with mouse fibroblasts transfected with human IR-B and with deleted mouse IGF-1R according to Zakova et al. ${ }^{50}$ Representative binding curves of analogues with the receptors are shown in Figure S1 (IGF-1R), Figure S2 (IR-A), and Figure S3 (IR-B). The binding curve of each analogue was determined in duplicate, and the final dissociation constant $\left(K_{\mathrm{d}}\right)$ was calculated from at least three $(n$ $\geq 3$ ) binding curves ( $K_{d}$ values), determined independently, and compared to binding curves for IGF-1 or for IGF-2, depending on the type of analogue.

The abilities of analogues to induce autophosphorylation of IGF-1R in membranes of mouse fibroblasts transfected with human IGF-1R and with deleted mouse IGF-1R were determined, as described by Machackova et al. ${ }^{51}$ The abilities of analogues to induce autophosphorylation of IR-A in mouse fibroblasts transfected with human IR-A and with deleted mouse IGF-1R were determined, as described by Krizkova et al. ${ }^{52}$ Briefly, the cells were stimulated in 24-well plates (Schoeller) $\left(4 \times 10^{4}\right.$ cells per well $)$ after being starved for 4 $\mathrm{h}$ in serum-free medium. The cells were stimulated with $10 \mathrm{nM}$ ligand (insulin, IGF-1, IGF-2, or analogues) for $10 \mathrm{~min}$. Stimulation was stopped by snap-freezing. Proteins were routinely analyzed, using immunoblotting and horseradish peroxidase-labeled secondary antibodies (Sigma-Aldrich). The membranes were probed with anti-phospho-IGF-1R $\beta$ (Tyr1135/1136)/IR $\beta$ (Tyr1150/1151) (Cell Signaling Technology). The blots were developed using the SuperSignal West Femto maximum sensitivity substrate (Pierce) and analyzed using the ChemiDoc MP Imaging System (Bio-Rad). The autophosphorylation signal density generated by each ligand on a Western blot was expressed as the contribution of phosphorylation relative to the IGF-1 (IGF-1R fibroblasts) respective human insulin (IR-A fibroblasts) signal in the same experiment. Mean \pm standard deviation (SD) values were calculated from four independent experiments $(n=4)$ and compared to those of native IGF-1 or native IGF-2, depending on the type of analogue. A representative example of an immunoblot used for the evaluation of the abilities of analogues to induce autophosphorylation of receptors is shown in Figure S4.

The dose-response curves for human IGF-1 and [His45]IGF-1, [Asn45]-IGF-1, [Ala45]-IGF-1, [His46]-IGF-1, and [Gln46]-IGF-1 analogues were also measured to determine their $\mathrm{EC}_{50}$ values and their abilities to stimulate the autophosphorylation of IGF-1R; here, the same methodology as for the measurements at a single dose (above) was followed. Log(agonist) versus response (variable slope) curve fitting of data was performed with GraphPad Prism 5. The representative curves are shown in the Figure S5. The $\mathrm{EC}_{50}$ values (calculated from at least three independent curves) are shown in the Table S2.

The significance of the changes in binding affinities and in the abilities of analogues to stimulate autophosphorylation was calculated using the two-tailed $t$ test.

\section{RESULTS}

Production of IGF-1 and IGF-2 Analogues. The production of IGF-1 and IGF-2 was achieved by their 
recombinant expression in Escherichia coli as a fusion with an $\mathrm{N}$ terminal and cleavable His6-tagged GB1 protein (immunoglobulin binding domain B1 of streptococcal protein-G), followed by the cleavage of the fusion protein with TEV protease. Recently, we used this strategy for the synthesis of IGF-2 analogues modified in the hormone's $\mathrm{C}$ domain and possessing an extra glycine residue at their $\mathrm{N}$-terminus (position -1$)^{40}$ Here, we modified the TEV cleavage site (see Materials and Methods) and succeeded in producing native IGF-2 without the additional Gly-1.

However, this strategy was not successful for IGF-1 because of the proline residue at position 2 in IGF-1 (Figure 1), which hampered TEV protease-mediated cleavage. Therefore, all analogues of the hormone produced in this work have an extra glycine residue $(\mathrm{Gly}-1)$ at the protein $\mathrm{N}$-terminus that enabled TEV protease cleavage of the precursor. The presence of Gly-1 did not have any significant effect on the binding properties of the IGF-1 derivative for either tested receptor [IGF-1R or IR-A (Table 1 or 2, respectively)], and both native IGF-1 and Gly-1-IGF-1 can be considered as equipotent.

Design of the First Series of Analogues. The first series of analogues was designed with the substitution of IGF-1 Asp45 with "insulin-inspired" His, Asn, and Ala, and a similar strategy was applied for the replacement of IGF-1 Glu46 with His, Gln, and Ala. The Asn 45 and Gln46 mutations were also combined. All planned IGF-1 analogues (Tables 1 and 2) were successfully produced in quantities sufficient for their biological and physicochemical characterization.

In parallel, similar mutations were designed for IGF-2 at positions Glu44 and Glu45 that correspond to IGF-1 positions 45 and 46 (Figure 1). However, only one IGF-2 analogue, [Gln45]-IGF-2, was made with a significant yield that allowed its characterization. All other IGF-2 analogues formed insoluble precipitates after the TEV cleavage/folding steps.

IGF-1R Binding and Activation Properties of IGF-1 Analogues Modified at Positions 45 and 46, and the [GIn45]-IGF-2 Analogue. The analogues were tested for their binding to the IGF-1 receptor (IGF-1R) (Table 1), and their binding data were compared with the abilities of the analogues to induce autophosphorylation of the IGF-1R at a concentration of $10 \mathrm{nM}$ (Figure 3A). For human IGF-1 and [His45]IGF-1, [Asn45]-IGF-1, [Ala45]-IGF-1, [His46]-IGF-1 and [Gln46]-IGF-1 analogues, we also determined $\mathrm{EC}_{50}$ values of their abilities to stimulate autophosphorylation of IGF-1R (Figure S5).

In general, the mutations did not dramatically alter the binding characteristics of the analogues in comparison with those of native IGFs. However, it can be noted that analogues with mutations at site 45, [His45]-IGF-1, [Asn45]-IGF-1, and [Ala45]-IGF-1, have significantly reduced (29-60\%) binding affinities for IGF-1R, with the lowest values being that of [Ala45]-IGF-1.

The only successfully prepared IGF-2 analogue in this series, [Gln45]-IGF-2, had reduced binding potency for IGF-1R compared to that of native IGF-2 (Table 1) but activated IGF1R like native IGF-2 did (Figure 3A).

Relative $\mathrm{EC}_{50}$ values of IGF-1R stimulation by the selected analogues were in good general agreement with their relative ability to stimulate this receptor performed at a set ligand concentration of $10 \mathrm{nM}$ (Table S2). Hence, it appeared that the autophosphorylation abilities of hormones determined at their $10 \mathrm{nM}$ concentrations were good representations of their properties, and as this approach substantially improved the time
Table 1. IGF-1R Binding Affinities of Native Hormones and Analogues Reported in This Work ${ }^{a}$

\begin{tabular}{|c|c|c|}
\hline analogue & $\begin{array}{c}K_{\mathrm{d}} \pm \mathrm{SD}(\mathrm{nM})(n) \text { for } \\
\text { the human IGF-1R in } \\
\text { mouse fibroblasts }\end{array}$ & $\begin{array}{c}\text { relative binding } \\
\text { affinity for human } \\
\text { IGF-1R (\%) relative } \\
\text { to that of human } \\
\text { IGF-1 }\end{array}$ \\
\hline \multirow[t]{5}{*}{ human IGF-1 } & $0.24 \pm 0.05^{b}(5)$ & $100 \pm 21$ \\
\hline & $0.12 \pm 0.01^{c}(5)$ & $100 \pm 8$ \\
\hline & $0.34 \pm 0.12^{d}(4)$ & $100 \pm 35$ \\
\hline & $0.16 \pm 0.06^{e}(3)$ & $100 \pm 37$ \\
\hline & $0.25 \pm 0.03^{f}(4)$ & $100 \pm 12$ \\
\hline \multicolumn{3}{|l|}{ first series of IGF-1 analogues } \\
\hline Gly-1-IGF-1 & $0.25 \pm 0.02^{b}$ & $96 \pm 8$ \\
\hline [His45]-IGF-1 & $0.39 \pm 0.11^{c}(4)^{* * *}$ & $31 \pm 9$ \\
\hline [Asn45]-IGF-1 & $0.20 \pm 0.07^{c}(3)^{*}$ & $60 \pm 21$ \\
\hline [Ala45]-IGF-1 & $0.41 \pm 0.27^{c}(4)^{*}$ & $29 \pm 19$ \\
\hline [His46]-IGF-1 & $0.18 \pm 0.04^{b}(4)$ & $133 \pm 29$ \\
\hline$[\mathrm{G} \ln 46]-\mathrm{IGF}-1$ & $0.18 \pm 0.01^{b}(3)$ & $133 \pm 7$ \\
\hline [Ala46]-IGF-1 & $0.26 \pm 0.11^{b}(3)$ & $92 \pm 39$ \\
\hline$[$ Asn45,Gln46]-IGF-1 & $0.70 \pm 0.28^{d}(4)$ & $49 \pm 20$ \\
\hline \multicolumn{3}{|l|}{ second series of IGF-1 analogues } \\
\hline [His49]-IGF-1 & $0.50 \pm 0.23^{d}(4)$ & $68 \pm 31$ \\
\hline [His46,His49]-IGF-1 & $0.29 \pm 0.17^{e}(4)$ & $55 \pm 32$ \\
\hline [Gln46,His49]-IGF-1 & $0.30 \pm 0.09^{e}(3)$ & $53 \pm 16$ \\
\hline $\begin{array}{l}\text { [Asn45,Gln46,His49]- } \\
\text { IGF-1 }\end{array}$ & $0.92 \pm 0.04^{d}(3) * * *$ & $37 \pm 2$ \\
\hline human IGF-2 & $2.3 \pm 1.2^{f}(3)^{*}$ & $10.9 \pm 5.7$ \\
\hline \multicolumn{3}{|l|}{ first series of IGF-2 analogues } \\
\hline$[\mathrm{G} \ln 45]-\mathrm{IGF}-2$ & $2.9 \pm 0.3^{e}(3)^{*}$ & $5.5 \pm 0.6$ \\
\hline \multicolumn{3}{|l|}{ second series of IGF-2 analogues } \\
\hline [His48]-IGF-2 & $0.88 \pm 0.23^{e}(3)$ & $18.2 \pm 4.7$ \\
\hline [Gln45,His48]-IGF-2 & $0.89 \pm 0.05^{e}(3)$ & $18.0 \pm 1.0$ \\
\hline
\end{tabular}

${ }^{a}$ The values of $K_{\mathrm{d}}$ and relative binding affinities [relative receptor binding affinity defined as $\left(K_{\mathrm{d}}\right.$ of human IGF-1)/( $K_{\mathrm{d}}$ of analogue $) \times$ 100] of human IGF-1, IGF-2, and analogues were determined for human IGF-1R in mouse fibroblasts. All IGF-1 analogues have an extra glycine residue at the $\mathrm{N}$-terminus $(\mathrm{Gly}-1) . n$ is the number of replicates. Asterisks indicate that binding of a particular ligand to IGF$1 \mathrm{R}$ differs significantly $(* p<0.05 ; * * *<0.001)$ from the effect of IGF-1 in the case of IGF-1 analogues or differs significantly from the effect of IGF-2 in the case of IGF-2 analogues. Binding of native IGF-2 is related to that of human IGF-1. ${ }^{b}$ Relative to the human IGF-1 $K_{\mathrm{d}}$ value of $0.24 \pm 0.05(n=5)$. ${ }^{c}$ Relative to the human IGF-1 $K_{d}$ value of $0.12 \pm 0.01(n=5) .{ }^{d}$ Relative to the human IGF-1 $K_{\mathrm{d}}$ value of $0.34 \pm$ $0.12(n=4) .{ }^{e}$ Relative to the human IGF-1 $K_{\mathrm{d}}$ value of $0.16 \pm 0.06(n$ = 3). ${ }^{f}$ Relative to the human IGF-1 $K_{\mathrm{d}}$ value of $0.25 \pm 0.03(n=4)$.

and material economy of this extensive methodology, the receptor activation abilities of the rest of the analogues were measured at this set ligand concentration only. In general, no major discrepancies between the IGF-1R binding and activation properties of the analogues mutated at positions 45 and 46 of IGF-1 and [Gln45]-IGF-2 were observed (Table 1 and Figure 3A). Some analogues, e.g., [His46]-IGF-1 and [Gln46]-IGF-1, activated IGF-1R slightly less strongly than human IGF-1 did, but their apparent higher binding affinities for this receptor were not statistically significant. In contrast, [His45]-IGF-1, [Asn45]-IGF-1, and [Ala45]-IGF-1 analogues activated IGF-1R like human IGF-1 did, but their binding affinities were significantly reduced. Therefore, although some minor discrepancies could be observed here, any clear and major antagonism, or receptor overstimulation, was not detected.

IR-A Binding and Activation Properties of IGF-1 Analogues Modified at Sites 45 and 46, and the 
Table 2. IR-A Receptor Binding Affinities of Native Hormones and Analogues Reported in This Work ${ }^{a}$

\begin{tabular}{|c|c|c|}
\hline analogue & $\begin{array}{c}K_{\mathrm{d}} \pm \mathrm{SE}(\mathrm{nM})(n) \text { for } \\
\text { human IR-A in IM-9 } \\
\text { lymphocytes }\end{array}$ & $\begin{array}{l}\text { relative binding } \\
\text { affinity for human } \\
\text { IR-A (\%) relative to } \\
\text { that of human } \\
\text { insulin }\end{array}$ \\
\hline \multirow[t]{5}{*}{ human insulin } & $0.25 \pm 0.05^{b}(5)$ & $100 \pm 20$ \\
\hline & $0.27 \pm 0.02^{c}(5)$ & $100 \pm 7$ \\
\hline & $0.18 \pm 0.01^{d}$ & $100 \pm 6$ \\
\hline & $0.32 \pm 0.09^{e}(4)$ & $100 \pm 28$ \\
\hline & $0.30 \pm 0.13^{f}(5)$ & $100 \pm 43$ \\
\hline human IGF-1 & $23.7 \pm 11.5^{b}(3) * * *$ & $1.1 \pm 0.5$ \\
\hline \multicolumn{3}{|l|}{ first series of IGF-1 analogues } \\
\hline Gly-1-IGF-1 & $35.6 \pm 11.9^{b}(3)$ & $0.7 \pm 0.2$ \\
\hline [His45]-IGF-1 & $20.1 \pm 7.8^{c}(4)$ & $1.3 \pm 0.5$ \\
\hline [Asn45]-IGF-1 & $19.3 \pm 9.6^{c}(4)$ & $1.4 \pm 0.7$ \\
\hline [Ala45]-IGF-1 & $17.6 \pm 9.7^{c}(3)$ & $1.5 \pm 0.8$ \\
\hline [His46]-IGF-1 & $6.6 \pm 1.2^{d}(3)$ & $2.7 \pm 0.5$ \\
\hline$[\mathrm{G} \ln 46]-\mathrm{IGF}-1$ & $18.1 \pm 3.6^{d}(3)$ & $1.0 \pm 0.2$ \\
\hline [Ala46]-IGF-1 & $14.0 \pm 1.9^{d}(3)$ & $1.3 \pm 0.2$ \\
\hline$[$ Asn 45,Gln46]-IGF-1 & $17.5 \pm 8.4^{e}$ & $1.8 \pm 0.9$ \\
\hline \multicolumn{3}{|l|}{ second series of IGF-1 analogues } \\
\hline [His49]-IGF-1 & $6.7 \pm 2.4^{e}(3)^{*}$ & $4.8 \pm 1.7$ \\
\hline [His46,His49]-IGF-1 & $3.4 \pm 1.7^{e}(3)^{*}$ & $9.4 \pm 4.7$ \\
\hline [Gln46,His49]-IGF-1 & $7.5 \pm 4.1^{e}(3)^{*}$ & $4.3 \pm 2.3$ \\
\hline $\begin{array}{l}{[\text { Asn45,Gln46,His49]- }} \\
\text { IGF-1 }\end{array}$ & $5.5 \pm 2.5^{f}(4)^{* * *}$ & $5.5 \pm 2.5$ \\
\hline human IGF-2 & $2.9 \pm 0.2^{b}(3)^{* * *}$ & $8.6 \pm 0.6$ \\
\hline \multicolumn{3}{|l|}{ first series of IGF- 2 analogues } \\
\hline$[\mathrm{G} \ln 45]-\mathrm{IGF}-2$ & $1.6 \pm 0.3^{e}(3)^{* *}$ & $20 \pm 3.8$ \\
\hline \multicolumn{3}{|l|}{ second series of IGF- 2 analogues } \\
\hline [His48]-IGF-2 & $0.54 \pm 0.13^{e}(3)^{* * *}$ & $59.3 \pm 14.3$ \\
\hline [Gln45,His48]-IGF-2 & $0.65 \pm 0.12^{f}(3) * * *$ & $46.2 \pm 8.5$ \\
\hline
\end{tabular}

${ }^{a}$ The values of $K_{\mathrm{d}}$ and relative binding affinities [relative receptor binding affinity defined as $\left(K_{\mathrm{d}}\right.$ of human insulin $) /\left(K_{\mathrm{d}}\right.$ of analogue $) \times$ $100]$ of human insulin, IGF-1, IGF-2, and analogues were determined for human IR-A in human IM-9 lymphocytes. All IGF-1 analogues have an extra glycine residue at the $\mathrm{N}$-terminus (Gly-1). $n$ is the number of replicates. Asterisks indicate that binding of a particular ligand to IR-A differs significantly $(* p<0.05$; $* p<0.01$; $* * p<$ 0.001 ) from the effect of IGF-1 in the case of IGF-1 analogues or differs significantly from the effect of IGF-2 in the case of IGF-2 analogues. Binding of native IGF-2 is related to that of human insulin. ${ }^{b}$ Relative to the human insulin $K_{\mathrm{d}}$ value of $0.25 \pm 0.05(n=5)$. ${ }^{c}$ Relative to the human insulin $K_{d}$ value of $0.27 \pm 0.02(n=5)$. ${ }^{d}$ Relative to the human insulin $K_{\mathrm{d}}$ value of $0.18 \pm 0.01(n=4)$. ${ }^{e}$ Relative to the human insulin $K_{\mathrm{d}}$ value of $0.32 \pm 0.09(n=4)$.

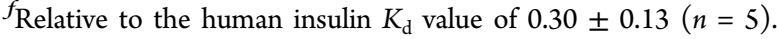

[GIn45]-IGF-2 Analogue. The IGF-1 analogues of the first series have binding affinities for IR-A similar to that of native IGF-1 (Table 2), and their IR-A activation properties (Figure 3B) are again in general agreement with the properties of native IGF-1.

The [Gln45]-IGF-2 analogue binds IR-A significantly more strongly ( $\leq 20 \%$ of the binding affinity of native human insulin) than the native IGF- 2 that has only $8 \%$ of the binding affinity of human insulin. However, the IR-A enhanced affinity of this analogue was not fully translated into its activation potency that is similar to the activation potency of native IGF-2.

Design of the Second Series of IGF-1 and IGF-2 Analogues. The ThrA8His substitution in insulin increases the potency for IR-A; ${ }^{41-46}$ hence, it was also used by Whittaker et al. ${ }^{37}$ to increase the level of IR binding of A4/A5-modified
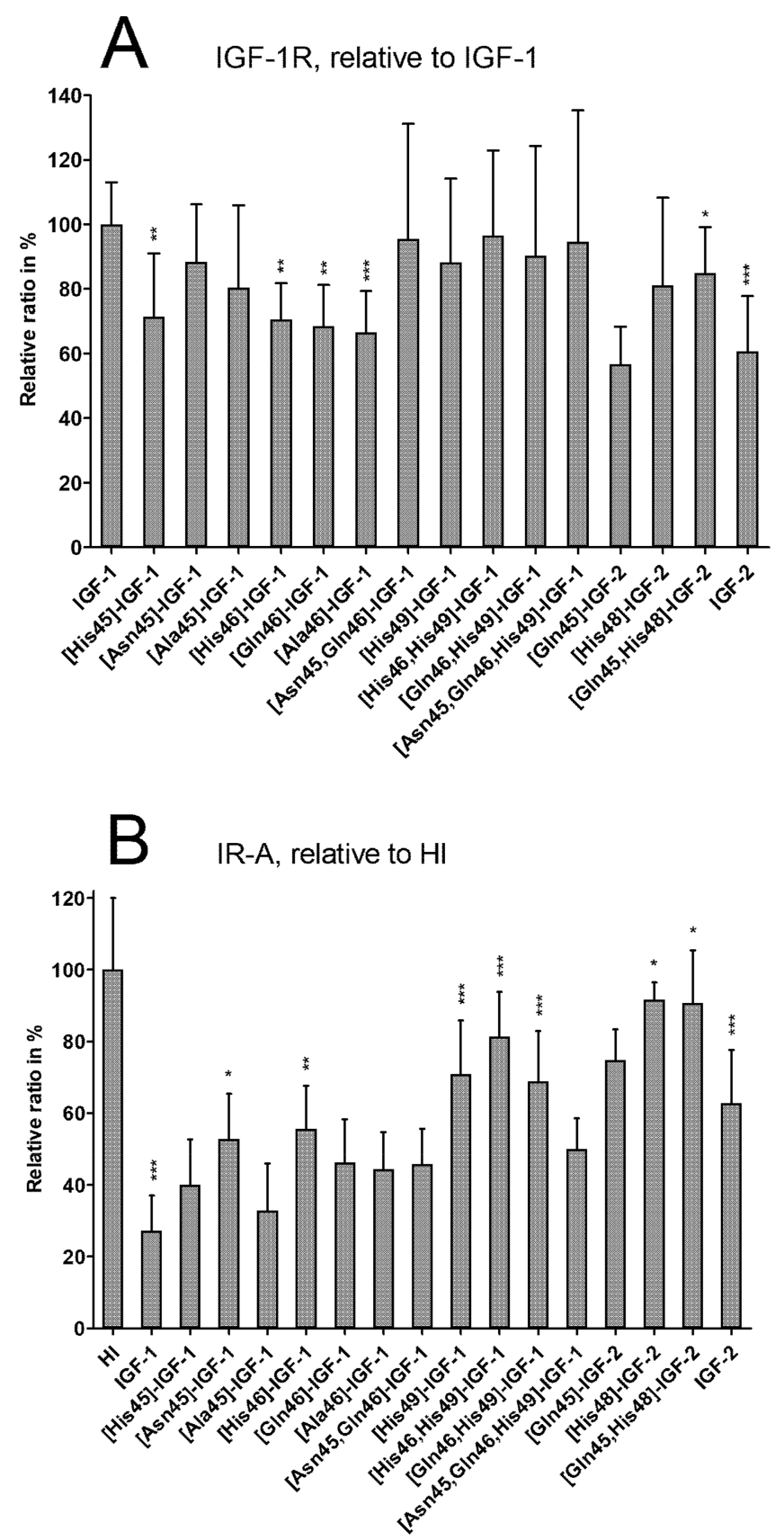

Figure 3. Relative abilities to activate (A) IGF-1R and (B) IR-A of human insulin (HI), human IGF-1, human IGF-2, and IGF-1 and IGF2 analogues. All IGF-1 analogues contain a glycine residue at position -1 . Relative abilities to activate receptors were determined with $10 \mathrm{nM}$ ligands after a $10 \mathrm{~min}$ stimulation. Mean $\pm \mathrm{SD}$ values were calculated from four independent experiments $(n=4)$. In panel $A$, the experimental values are related to the biological activity of human IGF-1. In panel B, the experimental values are the biological activity of human insulin (HI). Asterisks indicate that induction of autophosphorylation of a particular receptor induced by a ligand differs significantly $(* p<0.05 ; * *<0.01 ; * * * p<0.001)$ from the effect of IGF-1 in the case of IGF-1 analogues or differs significantly from the effect of IGF-2 in the case of IGF-2 analogues. In panel A, the significance of the effect of native IGF-2 (asterisks) is related to human IGF-1 and in panel B to human insulin. In panel B, the significance of the effect of native IGF-1 (asterisks) is related to human insulin.

insulins, without eliminating their antagonistic properties. Therefore, we probed a similar strategy for the IGF-1 and 
IGF-2 analogues, which both have phenylalanine at the insulin A8 equivalent 49 and 48 sites, respectively (Figure 1).

First, the [His49]-IGF-1 analogue was made to investigate the effect of this single mutation. Subsequently, it was combined with His or Gln single mutations at site 46 and with Asn45/Gln46 double mutations, as well.

In the case of IGF-2, the single mutation [His48]-IGF-2 analogue was made, which was extended for Gln 45 mutation, as well, as it was here the only successful substitution of IGF-2 in the first series.

IGF-1R Binding and Activation Properties of the Second Series of IGF-1 and IGF-2 Analogues. All new IGF-1 mutations have fairly minor, or no significant, effects on IGF-1R binding affinities and activation capabilities, with only the [Asn45,Gln46,His49]-IGF-1 triple mutant having significantly less affinity (37\%) for the IGF-1R than native IGF-1 has but with native IGF-1-like autophosphorylation activation ability (Table 1 and Figure 3A).

A similar trend was observed for both new IGF-2 analogues, [His48]-IGF-2 and [Gln45,His48]-IGF-2, the IGF-1R binding and activation abilities of which were similar to those of native IGF-2.

IR-A Binding and Activation Properties of the Second Series of IGF-1 and IGF-2 Analogues. The IR-A-related properties of the analogues contrast with their IGF-1R affinities and binding effects. It seems that the presence of His49 strongly enhances (4-9-fold) the IR-A binding affinity of new IGF-1 analogues (Table 2), in comparison to that of native IGF-1. Moreover, His49-containing IGF-1 analogues do not show any IR-A antagonism, as their capabilities to activate this receptor are superior, or similar, to those of native IGF-1 (Figure 3B).

IR-A binding affinities of two new IGF-2 analogues carrying a His48 mutation are also very (5-7-fold) enhanced, in comparison with those of native IGF-2. Remarkably, both of these analogues bind IR-A with subnanomolar affinities, which make them half-equipotent with human insulin with respect to this IR isoform (Table 2). Moreover, the IR-A activation abilities of these two IGF-2 analogues are also very high, with a potency similar to that of human insulin (Figure 3B).

In general, the levels of activation of IR-A by insulin, IGF-1, IGF-2, and analogues determined at $10 \mathrm{nM}$ (Figure 3B) correspond well to their respective binding affinities for this receptor (Table 2), and they are in a good agreement with the levels of IR-A autophosphorylation induced by $10 \mathrm{nM}$ insulin, IGF-1, and IGF-2 in dose-response curves reported recently by Andersen et al. ${ }^{53}$

IR-B Binding Properties of [His49]-IGF-1 and [Gln45,His48]-IGF-2 Analogues. The outstanding enhancement of IR-A binding affinities exhibited by the His49/His48 IGF-1/2 mutants prompted their characterization toward the IR-B isoform, as well. For this purpose, we tested two representative analogues, [His49]-IGF-1 and [Gln45,His48]IGF-2, for their binding affinity for IR-B (Table 3). Most interestingly, we found that the IR-B binding affinity of [Gln45,His48]-IGF-2 is $\sim 8$ times higher than that of human IGF-2 and that the binding of [His49]-IGF-1 to this IR isoform is $\sim 3$ times stronger than that of native IGF-1. These mutations did not considerably affect the IR isoform specificity of the analogues because their IR-A binding enhancing effects were similar (5 times for [Gln45,His48]-IGF-2 and $\sim 4$ times for [His49]-IGF-1).
Table 3. IR-B Receptor Binding Affinities of Native Hormones and [His49]-IGF-1 and [Gln45,His48]-IGF-2 Analogues Reported in This Work ${ }^{a}$

$\begin{array}{lcc}\text { analogue } & \begin{array}{c}K_{\mathrm{d}} \pm \mathrm{SE}(\mathrm{nM})(n) \text { for } \\ \text { human IR-B in mouse } \\ \text { fibroblasts }\end{array} & \begin{array}{c}\text { relative binding affinity for } \\ \text { human IR-B }(\%) \text { relative to that } \\ \text { of human insulin }\end{array} \\ \text { human insulin } & 0.50 \pm 0.31(5) & 100 \pm 62 \\ \text { human IGF-1 } & 224 \pm 16(4)^{b_{* * *}} & 0.22 \pm 0.02 \\ \text { [His49]-IGF-1 } & 72.3 \pm 12.0(3)^{* * *} & 0.69 \pm 0.11 \\ \text { human IGF-2 } & 35.5 \pm 5.6(4)^{b_{* * *}} & 1.4 \pm 0.2 \\ \text { [Gln45,His48]- } & 4.3 \pm 1.7(4)^{* *} & 11.6 \pm 4.6 \\ \text { IGF-2 } & & \end{array}$

${ }^{a_{T}}$ The values of $K_{\mathrm{d}}$ and relative binding affinities [relative receptor binding affinity defined as $\left(K_{\mathrm{d}}\right.$ of human insulin $) /\left(K_{\mathrm{d}}\right.$ of analogue $) \times$ $100]$ of human insulin, IGF-1, IGF-2, and analogues were determined for human IR-B in mouse fibroblasts. $n$ is the number of replicates. Asterisks indicate that the binding a particular ligand to IR-B differs significantly $(* * p<0.01$; $* * * p<0.001)$ from the effect of IGF-1 in the case of an IGF-1 analogue or differs significantly from the effect of IGF-2 in the case of an IGF-2 analogue. The binding of native IGF-1 and IGF-2 is relative to that of human insulin. ${ }^{b}$ From ref 52.

\section{DISCUSSION}

All 11 planned IGF-1 analogues were successfully produced, but only three IGF-2 analogues ([Gln45]-IGF-2, [Gln45,His49]-IGF-2, and [His49]-IGF-2) were made, as the other analogues of this hormone formed insoluble precipitates during the folding steps of their purification. As Whittaker et al. ${ }^{37}$ successfully prepared HisA4, AlaA4, and HisA5 insulins, the severe aggregation of some IGF-2 analogues indicates different folding mechanisms of these hormones, underlining the importance of IGF-2 Glu44/45 for its efficient assembly. However, a more extensive mutagenesis, especially of the IGF-2 Glu44 site, is needed for an unambiguous confirmation of the folding-related importance of these side chains.

In general, the majority of mutations performed here have a rather minor impact on the IGF-1R binding affinities of IGF-1 and IGF-2 analogues (Table 1). However, different trends may be observed for the His mutation at position 48 of IGF- 2 that enhances its IGF-1R affinity, while the His mutation at IGF-1 equivalent site 49 has an opposite effect. This may indicate that the natures of interactions of IGF-1 and IGF-2 with IGF-1R are different, and specific, for IGF-1 and IGF-2 at their sites 49 and 48 , respectively; i.e., the equivalent amino acids at these sites do not interact with the IGF-1R in the same fashion.

Interestingly, the mutations of sites 49 and 48 in IGF- 1 and IGF-2, respectively, yielded analogues of these hormones with much more significant, and interesting, changes in their IR-A binding affinities (Table 2) than in their IGF-1R binding/ activation properties. Here, both IGF-1 and IGF-2 analogues with His at sites 49 and 48, respectively, are much better IR-A binders than their native forms. The data presented here indicate that this effect can be attributed mainly to the isolated impact of His49, or His48, as the simultaneous mutations at these sites and of residues 45 and 46 in IGF-1 (or residue 45 in IGF-2) do not generate any additional, significant positive properties toward IR-A.

It has been shown that the ThrA8His mutation doubles or triples the insulin IR-A binding affinity, ${ }^{41-45}$ and Whittaker et al. ${ }^{37}$ used such a mutation to restore IR binding affinity of the less active A4 and A5 insulin mutants. Here, similar mutations enhanced 4-9-fold the IR-A binding affinities of the IGF-1 analogues and 5-7-fold the binding affinities of the IGF-2 
analogues, compared to the affinities of the native hormones. The positive effect of His49 and His48 mutations on IR-A binding is similar for both IGF-1 and IGF-2, and to the best of our knowledge, these analogues are the strongest IGF-1-like and IGF-2-like binders of the IR-A receptor isoform thus far reported. In particular, the high IR-A binding affinity of [His48]-IGF-2 is remarkable, as only this single mutation was sufficient to generate an analogue with $\sim 50 \%$ insulin-like affinity for the IR-A. Moreover, we found that the IR-A binding affinity enhancing effect of His49 and His48 is manifested with the IR-B, as well, because the [His49]-IGF-1 analogue is an $~ 3$ fold stronger IR-B binder than native IGF-1 is and, remarkably, [Gln45,His48]-IGF-2 binds IR-B 8-fold more strongly than native IGF-2 does, having almost $12 \%$ of the binding affinity of human insulin. Therefore, it seems that [His49]-IGF-1 and [Gln45,His48]-IGF-2 analogues are the strongest reported IGF-1-like and IGF-2-like IR-B binders. The exceptional binding "promiscuity" of [His48]-IGF-2 toward IR-A and IR$B$ is outstanding and reveals how easily IGF-2 can be converted into a high-affinity ligand for IR receptors. This "ubiquitous" hormonal property of IGF-2 may evoke questions about its evolutionary origins, and it could be hypothesized that IGF-2 resembles the hypothetical evolutionary hormonal ancestor of the insulin/IGF axis more closely than insulin or IGF-1 does.

It is generally accepted that insulin and IGFs interact with their receptors through two main binding sites, sites 1 and 2 in the hormones, and sites $1^{\prime}$ and $2^{\prime}$, respectively, in the receptors. ${ }^{54}$ The nature of the interactions of site 1 in insulin and IGF-1 with site $1^{\prime}$ in IR-A and IGF-1R is relatively well characterized in the crystal structures of their complexes (Figure 2). However, structural details about hormone site 2receptor site $2^{\prime}$ interactions are still missing, and insulin amino acids, which determine its site 2, ThrA8, IleA10, SerA12, LeuA13, GluA17, HisB10, GluB13, and LeuA17, have been suggested through extensive mutagenesis studies. ${ }^{54}$

The different effects of His mutations in IGFs on binding affinities for IR-A, IR-B, and IGF-1R could mean that amino acids at positions 49 (IGF-1) and 48 (IGF-2) are engaged with significantly different protein environments in complexes with IR and IGF-1R. Although it is expected that Phe49 in IGF-1, Phe48 in IGF-2, and ThrA8 in insulin (all at site 2 of the hormones) interact with $2^{\prime}$ sites of the receptors, the possibility that the increase in the level of IR-A binding of [His48]-IGF-2 and [His49]-IGF-1 analogues may also result from an enhancement of some contacts with elements of IR site $1^{\prime}$ cannot be excluded (Figure 2). For example, His49 and His48 of IGFs could form double direct, or water-mediated, hydrogen bonds with Asp707 in the IR $\alpha$-CT segment and with Asn694 in IGF-1R, as well (Figure 2A), while insulin native ThrA8 is too short for such interactions. It is also possible that the native Phe49 and Phe48 in IGFs can contribute only some weak van der Waals intramolecular contacts with the IGF-1 Cys48-Cys6 disulfide bond and Val44 (IGF-2 Cys47-Cys9 and Val43). Hence, His49 and His48 mutations may provide much stronger directional contacts with both receptor sites $\left(1^{\prime}\right.$ and $\left.2^{\prime}\right)$, which seem to be more favorable for IR-A rather than for IGF-1R.

In general, our new IGF-1 and IGF-2 analogues did not show any considerable reversed trends between their affinities and receptor activation abilities (Tables 1 and 2, Figure 3A,B, and Figure S5). Therefore, the previously observed significant disproportionate binding and activation of insulin analogues mutated at A4 and A8 (ref 37) are probably specific and limited for insulin-IR interactions. It is possible that a different receptor activation behavior of insulin and IGF-1 (IGF-2) analogues mutated at positions A4 and A8 and positions 45, 46, and 49 (positions 45 and 48), respectively, may result from different interactions with specific amino acids at $2^{\prime}$ sites of the IR that are currently not yet determined (see the preceding discussion). Such dissimilar natures of hormone-receptor interfaces may lead to non-equivalent receptor binding mechanisms (as previously pertinently mentioned by Sohma et al. in ref 55) and their subsequent different impacts on signal transduction through the receptors and, ultimately, the activation of their tyrosine kinases.

As indicated above, some trends observed in the analogues described here can be also corroborated by the phylogeny of insulin-related hormones. For example, the insulin ThrA8containing A8-A10 region is considered as a hypervariable part of this hormone because of its significant sequence differences in mammals. ${ }^{56}$ However, the A8 site is the most invariable amino acid in the A8-A10 triad. For example, the well IR-A tolerated ( $87 \%$ binding affinity of $\mathrm{HI}$ ) AlaA8 occurs in cattle, sheep, and goat insulin. ${ }^{57}$ Although there is no mammalian insulin with His at site A8, this amino acid is frequently present at that position in fish, frog, and bird insulins. ${ }^{56}$ Also, HisA8 was proposed to be responsible for an $\sim 5$-fold higher binding affinity of chicken insulin for IR in human lymphocytes. ${ }^{41}$ Herring at al. $^{46}$ proposed that it is possible that a "loweraffinity" ThrA8 site in mammals emerged from the evolutionary optimization of the insulin receptor kinetics, which requires a decrease in insulin binding affinity in mammals that is dictated by a different route of insulin delivery. In more ancient vertebrates like birds, insulin is secreted differently, by the kidney, which leads to rapid metabolic changes. ${ }^{46}$ However, in mammals, the pancreas-to-liver portal vein-mediated pathway is the primary direction of insulin. In humans, the liver is the main glycemic-response organ with $>90 \%$ "metabolic" IR-B isoform and where insulin inhibits gluconeogenesis and glycogenolysis before reaching the peripheral tissues. It may be postulated then that to ensure an optimum liver:body distribution of insulin its IR binding affinity needs to be reduced in mammals, to achieve a more systemic glycemic response. ${ }^{46}$

All known naturally occurring variants of IGF-1 and IGF-2 (including avian) maintain Phe at their insulin ThrA8corresponding sites 49 and 48, respectively. Therefore, it may be assumed that the IR high-affinity inducing HisA8 mutation in insulin selected upon evolution was required for only rapid and immediate metabolic effects of insulin and was not needed for slower and long-lasting growth effects of IGFs. However, a high sensitivity of both IGFs to acquisition of His48 and His49 mutations may reflect their common evolutionary origins with insulin.

It will be interesting to further explore the findings of this report by testing specific amino acid substitutions at the A8 site of insulin, to maintain its potent IR binding but to decrease its affinity for IGF-1R. Such analogues could be useful for the safer treatment of diabetes, as some currently administered insulin derivatives (e.g., glargine) show higher IGF-1R affinity and hence, potentially, an increased risk of cancer. ${ }^{58-62}$

In summary, as we showed that respective positions in insulin and IGFs lead to non-equivalent IR-A and IGF-1R binding mechanisms of these hormones, this work opens new directions for the rational engineering of the hormonal components of the insulin/IGF system. 


\section{CONCLUSIONS}

We systematically investigated the receptor binding and receptor activation properties of IGF-1 and IGF-2 analogues modified at positions 45,46 , and 49 (IGF-1) and at positions 44, 45, and 48 (IGF-2). These modifications did not significantly affect the IGF-1R binding of these hormones. However, analogues with the Phe 49His mutation in IGF-1 and the Phe 48His mutation in IGF-2 have remarkably enhanced binding affinities for both IR-A and IR-B isoforms of the insulin receptor. Here, IGF-1 analogues with His at position 49 possess approximately 5-9\% of IR-A, and [His49]-IGF-1 possess $\sim 0.7 \%$ of the IR-B binding affinity of human insulin. Moreover, IGF-2 analogues with His at position 48 are approximately halfequipotent to human insulin in binding to IR-A, and [Gln45,His48]-IGF-2 has almost $12 \%$ of the insulin binding affinity for this "metabolic" isoform. The binding affinities of all analogues are in general proportionate to their abilities to activate IR and IGF-1R without any important discrepancies. This study revealed that IR and IGF-1R can contain specific sites, probably parts of so-called receptors' sites $2^{\prime}$, which can interact differently with insulin and with IGFs. These findings shed light on new, possible directions of rational engineering of insulin and IGFs toward more selective and receptor-specific analogues with medical applications.

\section{ASSOCIATED CONTENT}

\section{S Supporting Information}

The Supporting Information is available free of charge on the ACS Publications website at DOI: 10.1021/acs.biochem.7b01260.

Summary of mutagenesis strategies used for cloning of IGF-1 and IGF-2 analogues (Table S1), binding curves of native hormones and analogues with IGF-1R, IR-A, and IR-B receptors (Figures $\mathrm{S} 1-\mathrm{S} 3$, respectively), representative immunoblot for determination of receptor activation abilities (Figure S4), and dose-response curves for determination of $\mathrm{EC}_{50}$ values of IGF-1R activation abilities of selected analogues (Figure S5) and their respective $\mathrm{EC}_{50}$ values (Table S2) (PDF)

\section{AUTHOR INFORMATION}

\section{Corresponding Authors}

*Telephone: +420 220183 441. E-mail: jiracek@uochb.cas.cz.

*E-mail: zakova@uochb.cas.cz.

\section{ORCID}

Andrzej M. Brzozowski: 0000-0001-7426-8948

Jiř́i Jiráček: 0000-0003-3848-2773

\section{Present Address}

"J.R: Department of Biochemistry, Faculty of Chemistry, University of Belgrade, Studentski trg 12-16, Belgrade, Serbia.

\section{Funding}

This work was supported by Czech Science Foundation Grant 15-19018S (to L.Z.), by Medical Research Council Grant MR/ K000179/1 (to A.M.B.), and partly also by Medical Research Council Grant MR/R009066/1 (to A.M.B.). Institutional support was provided by Projects RVO 61388963 (for the Institute of Organic Chemistry and Biochemistry) and 68378050 (for the Institute of Molecular Genetics) of the Czech Academy of Sciences.

\section{Notes}

The authors declare no competing financial interest.

\section{REFERENCES}

(1) Chan, S. J., Nagamatsu, S., Cao, Q. P., and Steiner, D. F. (1992) Structure and evolution of Insulin and Insulin-Like Growth-Factors in Chordates. Prog. Brain Res. 92, 15-24.

(2) Chan, S. J., and Steiner, D. F. (2000) Insulin through the ages: phylogeny of a growth promoting and metabolic regulatory hormone. Am. Zool. 40, 213-222.

(3) McRory, J. E., and Sherwood, N. M. (1997) Ancient divergence of insulin and insulin-like growth factor. DNA Cell Biol. 16, 939-949.

(4) LeRoith, D., Kavsan, V. M., Koval, A. P., and Roberts, C. T. (1993) Phylogeny of the insulin-like growth-factors (IGFs) and receptors - a molecular approach. Mol. Reprod. Dev. 35, 332-338.

(5) Denley, A., Cosgrove, L. J., Booker, G. W., Wallace, J. C., and Forbes, B. E. (2005) Molecular interactions of the IGF system. Cytokine Growth Factor Rev. 16, 421-439.

(6) Mayer, J. P., Zhang, F., and DiMarchi, R. D. (2007) Insulin structure and function. Biopolymers 88, 687-713.

(7) Stewart, C. E. H., and Rotwein, P. (1996) Growth, differentiation, and survival: multiple physiological functions for insulin-like growth factors. Physiol. Rev. 76, 1005-1026.

(8) Clemmons, D. R. (2009) Role of IGF-I in skeletal muscle mass maintenance. Trends Endocrinol. Metab. 20, 349-356.

(9) Holly, J. M. P., and Perks, C. M. (2012) Insulin-like growth factor physiology. What we have learned from human studies. Endocrinol. Metab. Clin. North Am. 41, 249-263.

(10) Dynkevich, Y., Rother, K. I., Whitford, I., Qureshi, S., Galiveeti, S., Szulc, A. L., Danoff, A., Breen, T. L., Kaviani, N., Shanik, M. H., LeRoith, D., Vigneri, R., Koch, C. A., and Roth, J. (2013) Tumors, IGF-2, and hypoglycemia: insights from the clinic, the laboratory, and the historical archive. Endocr. Rev. 34, 798-826.

(11) Alberini, C. M., and Chen, D. Y. (2012) Memory enhancement: consolidation, reconsolidation and insulin-like growth factor 2. Trends Neurosci. 35, 274-283.

(12) Chen, D. Y., Stern, S. A., Garcia-Osta, A., Saunier-Rebori, B., Pollonini, G., Bambah-Mukku, D., Blitzer, R. D., and Alberini, C. M. (2011) A critical role for IGF-II in memory consolidation and enhancement,. Nature 469, 491-497.

(13) Lawrence, M. C., McKern, N. M., and Ward, C. W. (2007) Insulin receptor structure and its implications for the IGF-1 receptor. Curr. Opin. Struct. Biol. 17, 699-705.

(14) De Meyts, P. (2004) Insulin and its receptor: structure, function and evolution. BioEssays 26, 1351-1362.

(15) De Meyts, P., and Whittaker, J. (2002) Structure-Function Relationships of Insulin and Insulin-Like Growth Factor-I Receptor Binding. In Insulin and Related Proteins: Structure to Function and Pharmacology (Dieken, M. L., Federwisch, M., and De Meyts, P., Eds.) pp 131-149, Kluwer Academic Publishers, Dordrecht, The Netherlands.

(16) Belfiore, A., Frasca, F., Pandini, G., Sciacca, L., and Vigneri, R. (2009) Insulin receptor isoforms and insulin receptor/insulin-like growth factor receptor hybrids in physiology and disease. Endocr. Rev. $30,586-623$.

(17) Belfiore, A., Malaguarnera, R., Vella, V., Lawrence, M. C., Sciacca, L., Frasca, F., Morrione, A., and Vigneri, R. (2017) Insulin receptor isoforms in physiology and disease: an updated view. Endocr. Rev. 38, 379-431.

(18) Vienberg, S. G., Bouman, S. D., Sorensen, H., Stidsen, C. E., Kjeldsen, T., Glendorf, T., Sorensen, A. R., Olsen, G. S., Andersen, B., and Nishimura, E. (2011) Receptor-isoform-selective insulin analogues give tissue-preferential effects. Biochem. J. 440, 301-308.

(19) Jiracek, J., and Zakova, L. (2017) Structural perspectives of insulin receptor isoform-slective insulin analogs. Front. Endocrinol. 8, 167.

(20) Siddle, K. (2012) Molecular basis of signaling specificity of insulin and IGF receptors: neglected corners and recent advances. Front. Endocrinol. 3, 34.

(21) Siddle, K. (2011) Signalling by insulin and IGF receptors: supporting acts and new players. J. Mol. Endocrinol. 47, R1-R10. 
(22) Boucher, J., Kleinridders, A., and Kahn, C. R. (2014) Insulin receptor signaling in normal and insulin-resistant states. Cold Spring Harbor Perspect. Biol. 6, a009191.

(23) Hers, I., Vincent, E. E., and Tavare, J. M. (2011) Akt signalling in health and disease. Cell. Signalling 23, 1515-1527.

(24) Bedinger, D. H., and Adams, S. H. (2015) Metabolic, anabolic, and mitogenic insulin responses: A tissue-specific perspective for insulin receptor activators,. Mol. Cell. Endocrinol. 415, 143-156.

(25) Sacco, A., Morcavallo, A., Pandini, G., Vigneri, R, and Belfiore, A. (2009) Differential signaling activation by insulin and insulin-like growth factors I and II upon binding to insulin receptor isoform A. Endocrinology 150, 3594-3602.

(26) Slaaby, R., Schaffer, L., Lautrup-Larsen, I., Andersen, A. S., Shaw, A. C., Mathiasen, I. S., and Brandt, J. (2006) Hybrid receptors formed by insulin receptor (IR) and insulin-like growth factor I receptor (IGFIR) have low insulin and high IGF-1 affinity irrespective of the IR splice variant. J. Biol. Chem. 281, 25869-25874.

(27) Slaaby, R. (2015) Specific insulin/IGF1 hybrid receptor activation assay reveals IGF1 as a more potent ligand than insulin. Sci. Rep. 5, 7911.

(28) Clemmons, D. R. (2016) Role of IGF binding proteins in regulating metabolism. Trends Endocrinol. Metab. 27, 375-391.

(29) Firth, S. M., and Baxter, R. C. (2002) Cellular actions of the insulin-like growth factor binding proteins. Endocr. Rev. 23, 824-854.

(30) Kornfeld, S. (1992) Structure and function of the mannose 6phosphate insulin-like growth factor-II receptors. Annu. Rev. Biochem. 61, 307-330.

(31) Belfiore, A., and Malaguarnera, R. (2011) Insulin receptor and cancer. Endocr.-Relat. Cancer 18, R125-R147.

(32) Gallagher, E. J., and LeRoith, D. (2011) Minireview: IGF, insulin, and cancer. Endocrinology 152, 2546-2551.

(33) Tao, Y., Pinzi, V., Bourhis, J., and Deutsch, E. (2007) Mechanisms of disease: signaling of the insulin-like growth factor I receptor pathway-therapeutic perspectives in cancer. Nat. Clin. Pract. Oncol. 4, 591-602.

(34) Baserga, R. (2013) The decline and fall of the IGF-I receptor,. J. Cell. Physiol. 228, 675-679.

(35) Schaffer, L., Brissette, R. E., Spetzler, J. C., Pillutla, R. C., Ostergaard, S., Lennick, M., Brandt, J., Fletcher, P. W., Danielsen, G. M., Hsiao, K. C., Andersen, A. S., Dedova, O., Ribel, U., Hoeg-Jensen, T., Hansen, P. H., Blume, A. J., Markussen, J., and Goldstein, N. I. (2003) Assembly of high-affinity insulin receptor agonists and antagonists from peptide building blocks. Proc. Natl. Acad. Sci. U. S. A. $100,4435-4439$.

(36) Pillutla, R., Brissette, R., Blume, A. J., Schaffer, L., Brandt, J., Goldstein, N. I., Spetzler, J., Ostergaard, S., and Hansen, P. H. (2011) Insulin and IGF-1 Receptor Agonists and Antagonists. U.S. Patent 2011/0124556 A1.

(37) Whittaker, J., Whittaker, L. J., Roberts, C. T., Phillips, N. B., Ismail-Beigi, F., Lawrence, M. C., and Weiss, M. A. (2012) $\alpha$-Helical element at the hormone-binding surface of the insulin receptor functions as a signaling element to activate its tyrosine kinase. Proc. Natl. Acad. Sci. U. S. A. 109, 11166-11171.

(38) Menting, J. G., Whittaker, J., Margetts, M. B., Whittaker, L. J., Kong, G. K. W., Smith, B. J., Watson, C. J., Zakova, L., Kletvikova, E., Jiracek, J., Chan, S. J., Steiner, D. F., Dodson, G. G., Brzozowski, A. M., Weiss, M. A., Ward, C. W., and Lawrence, M. C. (2013) How insulin engages its primary binding site on the insulin receptor. Nature 493, 241-245.

(39) Menting, J. G., Lawrence, C. F., Kong, G. K. W., Margetts, M. B., Ward, C. W., and Lawrence, M. C. (2015) Structural congruency of ligand binding to the insulin and insulin/type 1 insulin-like growth factor hybrid receptors. Structure 23, 1271-1282.

(40) Hexnerova, R., Krizkova, K., Fabry, M., Sieglova, I., Kedrova, K., Collinsova, M., Ullrichova, P., Srb, P., Williams, C., Crump, M. P., Tosner, Z., Jiracek, J., Veverka, V., and Zakova, L. (2016) Probing receptor specificity by sampling the conformational space of the insulin-like growth factor II C-domain. J. Biol. Chem. 291, 2123421245 .
(41) Simon, J., Freychet, P., Rosselin, G., and Demeyts, P. (1977) Enhanced binding affinity of chicken insulin in rat-liver membranes and human lymphocytes - relationship to kinetic properties of hormone-receptor interaction. Endocrinology 100, 115-121.

(42) Kaarsholm, N. C., Norris, K., Jorgensen, R. J., Mikkelsen, J., Ludvigsen, S., Olsen, O. H., Sorensen, A. R., and Havelund, S. (1993) Engineering stability of the insulin monomer fold with application to structure-activity relationships. Biochemistry 32, 10773-10778.

(43) Weiss, M. A., Hua, Q. X., Jia, W., Nakagawa, S. H., Chu, Y. C., Hu, S. Q., and Katsoyannis, P. G. (2001) Activities of monomeric insulin analogs at position A8 are uncorrelated with their thermodynamic stabilities. J. Biol. Chem. 276, 40018-40024.

(44) Weiss, M. A., Wan, Z., Zhao, M., Chu, Y. C., Nakagawa, S. H., Burke, G. T., Jia, W., Hellmich, R., and Katsoyannis, P. G. (2002) Non-standard insulin design: structure-activity relationships at the periphery of the insulin receptor. J. Mol. Biol. 315, 103-111.

(45) Wan, Z., Xu, B., Huang, K., Chu, Y. C., Li, B., Nakagawa, S. H., Qu, Y., Hu, S. Q., Katsoyannis, P. G., and Weiss, M. A. (2004) Enhancing the activity of insulin at the receptor interface: crystal structure and photo-cross-linking of A8 analogues. Biochemistry 43, 16119-16133.

(46) Herring, R., Jones, R. H., and Russell-Jones, D. L. (2014) Hepatoselectivity and the evolution of insulin. Diabetes, Obes. Metab. $16,1-8$.

(47) Laible, M., and Boonrod, K. (2009) Homemade site directed mutagenesis of whole plasmids. J. Visualized Exp. 27, No. e1135.

(48) Heckman, K. L., and Pease, L. R. (2007) Gene splicing and mutagenesis by PCR-driven overlap extension. Nat. Protoc. 2, 924932.

(49) Vikova, J., Collinsova, M., Kletvikova, E., Budesinsky, M., Kaplan, V., Zakova, L., Veverka, V., Hexnerova, R., Avino, R. J. T., Strakova, J., Selicharova, I., Vanek, V., Wright, D. W., Watson, C. J., Turkenburg, J. P., Brzozowski, A. M., and Jiracek, J. (2016) Rational steering of insulin binding specificity by intra-chain chemical crosslinking. Sci. Rep. 6, 19431.

(50) Zakova, L., Kletvikova, E., Lepsik, M., Collinsova, M., Watson, C. J., Turkenburg, J. P., Jiracek, J., and Brzozowski, A. M. (2014) Human insulin analogues modified at the B26 site reveal a hormone conformation that is undetected in the receptor complex. Acta Crystallogr., Sect. D: Biol. Crystallogr. 70, 2765-2774.

(51) Machackova, K., Collinsova, M., Chrudinova, M., Selicharova, I., Picha, J., Budesinsky, M., Vanek, V., Zakova, L., Brzozowski, A. M., and Jiracek, J. (2017) Insulin-like growth factor 1 analogs clicked in the C domain: chemical synthesis and biological activities. J. Med. Chem. 60, 10105-10117.

(52) Krizkova, K., Chrudinova, M., Povalova, A., Selicharova, I., Collinsova, M., Vanek, V., Brzozowski, A. M., Jiracek, J., and Zakova, L. (2016) Insulin-insulin-like growth factors hybrids as molecular probes of hormone:receptor binding specificity. Biochemistry 55, 2903-2913.

(53) Andersen, M., Nørgaard-Pedersen, D., Brandt, J., Pettersson, I., and Slaaby, R. (2017) IGF1 and IGF2 specificities to the two insulin receptor isoforms are determined by insulin receptor amino acid 718 . PLoS One 12, e0178885.

(54) De Meyts, P. (2015) Insulin/receptor binding: the last piece of the puzzle? BioEssays 37, 389-397.

(55) Sohma, Y., Pentelute, B. L., Whittaker, J., Hua, Q. X., Whittaker, L. J., Weiss, M. A., and Kent, S. B. H. (2008) Comparative properties of insulin-like growth factor 1 (IGF-1) and [Gly7D-Ala]IGF-1 prepared by total chemical synthesis. Angew. Chem., Int. Ed. 47, $1102-1106$.

(56) Conlon, J. M. (2001) Evolution of the insulin molecule: insights into structure-activity and phylogenetic relationships. Peptides 22, $1183-1193$.

(57) Kristensen, C., Kjeldsen, T., Wiberg, F. C., Schaffer, L., Hach, M., Havelund, S., Bass, J., Steiner, D. F., and Andersen, A. S. (1997) Alanine scanning mutagenesis of insulin. J. Biol. Chem. 272, 1297812983.

(58) Kurtzhals, P., Schaffer, L., Sorensen, A., Kristensen, C., Jonassen, I., Schmid, C., and Trub, T. (2000) Correlations of receptor binding 
and metabolic and mitogenic potencies of insulin analogs designed for clinical use. Diabetes 49, 999-1005.

(59) Hemkens, L. G., Grouven, U., Bender, R., Gunster, C., Gutschmidt, S., Selke, G. W., and Sawicki, P. T. (2009) Risk of malignancies in patients with diabetes treated with human insulin or insulin analogues: a cohort study. Diabetologia 52, 1732-1744.

(60) Jonasson, J. M., Ljung, R., Talback, M., Haglund, B., Gudbjornsdottir, S., and Steineck, G. (2009) Insulin glargine use and short-term incidence of malignancies-a population-based followup study in Sweden. Diabetologia 52, 1745-1754.

(61) Colhoun, H. M. (2009) Use of insulin glargine and cancer incidence in Scotland: a studyfrom the Scottish Diabetes Research Network Epidemiology Group. Diabetologia 52, 1755-1765.

(62) Currie, C. J., Poole, C. D., and Gale, E. A. M. (2009) The influence of glucose-lowering therapies on cancer risk in type 2 diabetes,. Diabetologia 52, 1766-1777. 